\title{
Calibration of Equivalent Tire Cornering Stiffness for Vehicle Reference Model in ESP Based on Fminsearch Method
}

\author{
Li Zhanqi, Li Fei, Liu Quanzhou \\ China Automotive Technology \& Research Center \\ Dongli District, Tianjin, China Email: lizhanqi@catarc.ac.cn
}

\begin{abstract}
Keywords: fminsearch; equivalent tire corner stiffness; 2-DOF vehicle model; electronic stability control
\end{abstract}

\begin{abstract}
The 2-DOF vehicle model is generally used to be reference model of automotive electronic stability program (ESP), while the equivalent tire cornering stiffnesses of front and rear axles are key parameters to build it. A 2-DOF vehicle model is built, and a direct searching unconstrained nonlinear optimization method-Fminsearch is introduced for calibrating the tire cornering stiffnesses of front and rear axles simply and effectively.
\end{abstract}

\section{Introduction}

Automotive electronic stability control system need to judge the driver's turning intension according to steering wheel angle and further decide whether the vehicle is stable. Usually a simplified 2-DOF vehicle model, taking the front wheel steering angle obtained from hand wheel steering angle as input, is used to be reference model, which calculates vehicle yaw rate as the driver's turning intension [1,2].

The 2-DOF vehicle model ignores many systems such as suspension and steering system, etc. While these systems has large effects on vehicle steering properties, for example, suspension K\&C character affects the variation of toe-in angle and after that affects vehicle steering movement [3]. To represent vehicle steering properties accurately, the vector sum of actual wheel slip angle, toe-in angle and other related angles can be as equivalent wheel slip angle. Then well-reasoned, there appears the concept of equivalent tire corning stiffness that is just the tire corning stiffness used in 2 -DOF vehicle model. To build the 2-DOF vehicle model accurately, the equivalent tire corning stiffnesses of front and rear axles must be calibrated from steady-state cornering response of vehicle road-test or precise complex vehicle model such as veDYNA and dSPACE ASM simulation.

Fminsearch is a method of multidimensional unconstrained nonlinear minimization optimization, which uses Nelder-Mead simplex direct search algorithm. The fminsearch method can be used in MATLAB, and the local optimal values could only be obtained because it seeks the optimal value locally. To obtain the expected optimal result, appropriate initial estimate values should be given [4].

\section{2-DOF Vehicle Model}

The 2-DOF vehicle model whose schematic diagram is as shown in Fig.1 has only two freedom degrees of lateral and yaw motion, ignoring effects of suspension and other systems, and considers vehicle only doing planar motion parallel to ground, i.e. the roll angle, pitch angle and vertical displacement are zero. The model takes the front wheel steering angle as input and assumes that the vehicle travels at a constant velocity $\left(V_{x}\right)$.

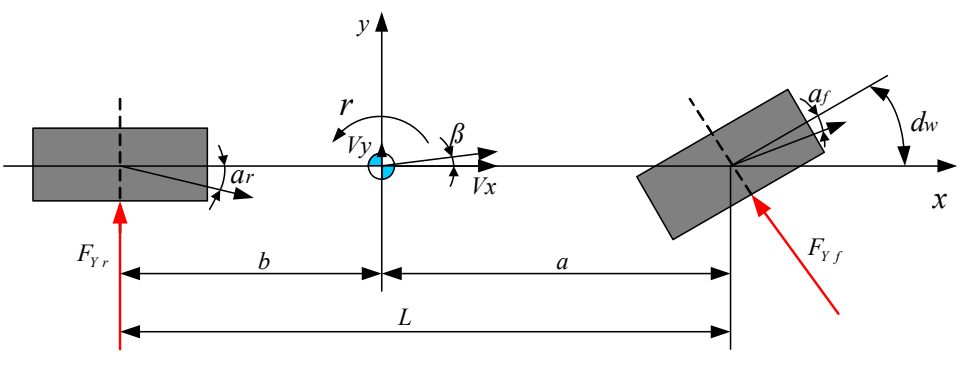

Figure 1. 2-DOF vehicle model schematic diagram

According to above hypothesis and d'Alembert principle, the balance equations along $y$-axis and rotation about $z$-axis list as follows: 


$$
\begin{aligned}
& \sum F_{y}=m \cdot\left(r \cdot V_{x}+\dot{V}_{y}\right), \\
& \sum M_{z}=I_{z} \cdot \dot{r} .
\end{aligned}
$$

The compositional forces present as follows:

$$
\begin{aligned}
\sum F_{y} & =F_{Y f}+F_{Y r}=k_{f} \cdot \alpha_{f}+k_{r} \cdot \alpha_{r}, \\
\sum M_{z} & =a \cdot F_{Y f}+b \cdot F_{Y r} \\
& =a \cdot k_{f} \cdot \alpha_{f}-b \cdot k_{r} \cdot \alpha_{r} .
\end{aligned}
$$

The positive direction of variables in above equations is in accordance with coordinate definition of Fig. 1. The front and rear tire cornering stiffnesses $\left(K_{f}, K_{\mathrm{r}}\right)$ are negative.

The sideslip angle $(\beta)$ is small when the vehicle stable [5] and $V_{x}$ is invariable, so there exist the following equations:

$$
\beta=V_{y} / V_{x} \quad \dot{\beta}=\dot{V}_{y} / V_{x} .
$$

The slip angles of front and rear wheel are given by:

$$
\begin{aligned}
& \alpha_{f}=-\delta_{w}+\beta+\frac{a \cdot r}{V_{x}}, \\
& \alpha_{r}=\beta-\frac{b \cdot r}{V_{x}} .
\end{aligned}
$$

In the above equations, $m$ is vehicle mass, $V_{x}$ is vehicle longitudinal velocity, $V_{y}$ is vehicle lateral velocity, $r$ is vehicle yaw rate, $I_{z}$ is moment of inertia around $z$ axis, $a$ is distance between the front axle and CG, $b$ is distance between the rear axle and CG, $\beta$ is vehicle sideslip angle, and $\delta_{w}$ is front wheel steering angle.

Based on Eq. (1) to (7), the state equations of 2-DOF vehicle model are obtained as follows:

$$
\left\{\begin{array}{l}
\dot{\beta}=\frac{A \cdot \beta+\left(B / V_{x}-m \cdot V_{x}\right) \cdot r-k_{f} \cdot \delta_{w}}{m \cdot V_{x}} \\
\dot{r}=\frac{B \cdot \beta+C \cdot r / V_{x}-k_{f} \cdot a \cdot \delta_{w}}{I_{z}}
\end{array}\right.
$$

Where:

$$
\left\{\begin{array}{l}
A=K_{f}+K_{r} \\
B=a \cdot K_{f}-b \cdot K_{r} \\
C=a^{2} \cdot K_{f}+b^{2} \cdot K_{r} .
\end{array}\right.
$$

Eq. (8) shows that 2-DOF vehicle model can describe the motion states of vehicle yaw rate and sideslip angle. The obtained yaw rate could be directly used to reflect the driver's steering intension, therefore, it is often called vehicle ideal yaw rate.

Known from Eq. (8) and (9), to accurately describe the vehicle ideal yaw rate, it is a key step to calibrate the equivalent tire cornering stiffness of front and rear axles $\left(K_{f}, K_{r}\right)$, in addition to know the given basic parameters of vehicle.

\section{Calibration of Equivalent Tire Cornering Stiffness of Front and Rear Axles with Fminsearch}

Selection of Optimization Parameters and Objective Function. Fminsearch is a method of 
multidimensional unconstrained nonlinear optimization. It finds the local minimum of a scalar function of several variables, starting at an initial estimate. Therefore, when using fminsearch method, firstly the optimization parameters should be determined and a initial estimate is given; then a appropriate objective function must be selected.

In this paper, the equivalent tire cornering stiffnesses of front and rear axles, $K_{f}, K_{r}$ are the optimization parameters of fminsearch function naturally.

In automotive electronic stability control system, the most purpose of 2-DOF vehicle model is to calculate vehicle ideal yaw rate to reflect the driver's steering intension. With vehicle lateral acceleration increasing, when tires enter into nonlinear region of cornering properties the vehicle actual yaw rate will deviate from the ideal value. If the deviation is large enough, ESP will intervene and make the vehicle actual yaw rate close to the ideal value [6]. When tires are in linear region of cornering properties, the vehicle is under control of the driver and the vehicle actual yaw rate is little different from the ideal value, i.e., the vehicle yaw rate obtained from 2-DOF vehicle model should be very nearly the same with the actual yaw rate as far as possible to avoid undesired ESP intervention [7]. So the sum of square of the difference between the vehicle yaw rate calculated from 2-DOF vehicle model and the actual yaw rate is selected as the objective function, and the vehicle tires should be in linear region of cornering properties when calibrating.

The objective function is given as:

$$
J=\int(r *-r)^{2} d t \quad \text { or } \quad J=\sum_{i=1}^{i=N}\left(r^{*}{ }_{i}-r_{i}\right)^{2}
$$

Where, $r^{*}$ and $r$ are respectively the vehicle yaw rate obtained from 2-DOF vehicle model and real car, taking the same front wheel steering angle as input, $N$ is the number of samples.

Calibration Process. In this paper, the high-precision dSPACE Automotive Simulation Model (ASM) is used to simulate the dynamic behavior of real vehicle. The calibration of $K_{f}$ and $K_{r}$ is done through comparing the yaw rate calculated from 2-DOF vehicle model with ASM under steering wheel angle step input maneuver when tires are in linear region of cornering properties.



Figure 2. Flow chart of calibrating $K_{f}, K_{r}$ with fminsearch method

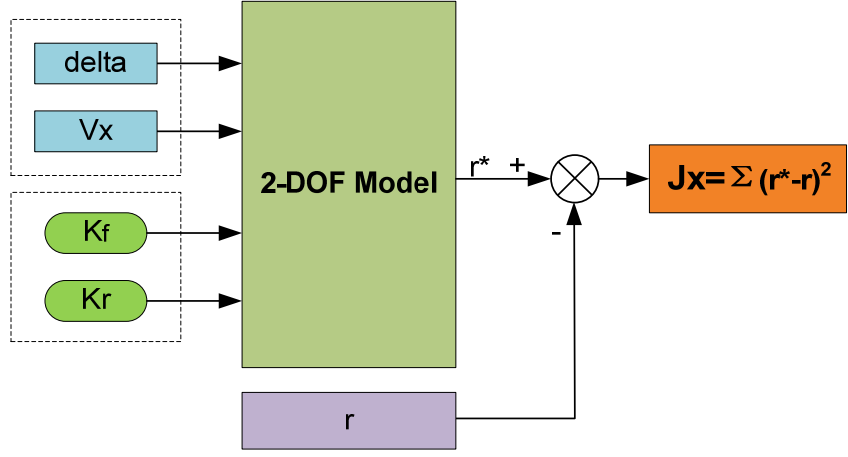

Figure 3. Block diagram of calculating fminsearch objective function

The detailed process of calibrating the equivalent tire cornering stiffnesses of front and rear axles is explained as follows (refer to Fig. 2): 
1) ASM simulates a set of vehicle yaw rate $(r)$ under given steering wheel angle step input and vehicle velocity.

2) In Simulink model, 2-DOF vehicle model calculates the ideal yaw rate $\left(r^{*}\right)$, taking the same steering angle (delta), vehicle velocity $(V x)$ as inputs and the given tire cornering stiffness $\left(K_{f}, K_{\mathrm{r}}\right)$ as parameters, (refer to Fig. 3). Then use $r^{*}$ minus $r$, and take the sum of square $\left(J_{x}=\Sigma\left(r^{*}-r\right)^{2}\right)$ of the difference as model output.

3) Fminsearch judges whether $J_{x}$ satisfy the appointed minimum requirement. If not, sets new values $\left(K_{f n}, K_{r \mathrm{n}}\right)$ of $K_{f}$ and $K_{\mathrm{r}}$ and continues next step calculation. If yes, outputs the objective values of the equivalent tire cornering stiffness of front and rear axles, meanwhile ends the simulation.

\section{Simulation Results and Validation}

In this paper, the object vehicle is a SUV model and the tire lateral force is in linear region.

Steering Wheel Angle Step Input. First, calibrate the equivalent tire cornering stiffnesses of front and rear axles under the vehicle velocity of $30 \mathrm{~km} / \mathrm{h}, 60 \mathrm{~km} / \mathrm{h}$ and $100 \mathrm{~km} / \mathrm{h}$ with fminsearch method, the result can be seen in Table 1; then substitute the respective calibration values into the 2-DOF vehicle model. The calibration is validated by comparing yaw rate of 2-DOF vehicle model with ASM under steering wheel angle step input maneuver.

Table 1. Equivalent Tire Cornering Stiffness

\begin{tabular}{c|c|c|c|c}
\hline \multicolumn{2}{l|}{ Vehicle Velocity $[\mathrm{km} / \mathrm{h}]$} & 30 & 60 & 100 \\
\hline Cornering Stiffness & $K_{f}$ (front) $^{*}$ & -80070 & -94180 & -94980 \\
\cline { 2 - 5 }$[\mathrm{N} / \mathrm{rad}]$ & $K_{r}{\text { (rear })^{*}}^{*}-109470$ & -135590 & -141270 \\
\hline
\end{tabular}

$* K_{f}$ is sum of two tire cornering stiffnesses of front axle; so as $K_{r}$.

Fig. 4 to 6 are steering wheel angle step input simulation results of ASM and 2-DOF vehicle model with respective equivalent tire cornering stiffnesses calibrated under different vehicle velocity.

Slalom Test. To further validate the accuracy of 2-DOF vehicle model in the linear region of tire cornering properties, slalom test with pile spacing of 40 meters is adopted following. This test proceeds under initial vehicle velocity of $70 \mathrm{~km} / \mathrm{h}$, refer to Fig.7. The equivalent tire cornering stiffnesses used are calibrated through steering wheel angle step input under vehicle velocity of 70 $\mathrm{km} / \mathrm{h}$; the calibration result is $K_{f}=-94320 \mathrm{~N} / \mathrm{rad}, K_{r}=-136380 \mathrm{~N} / \mathrm{rad}$.

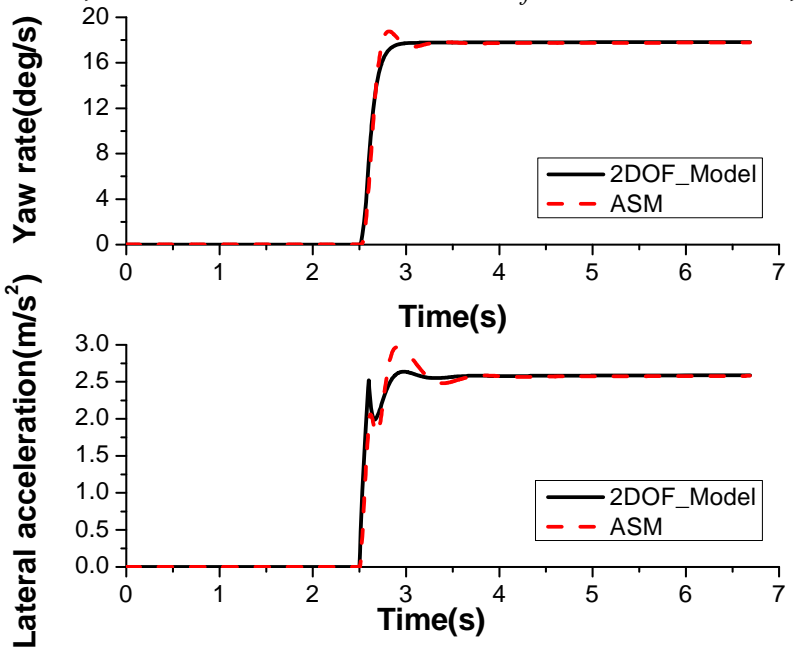

Figure 4. Steering wheel angle step input simulation $(30 \mathrm{~km} / \mathrm{h})$

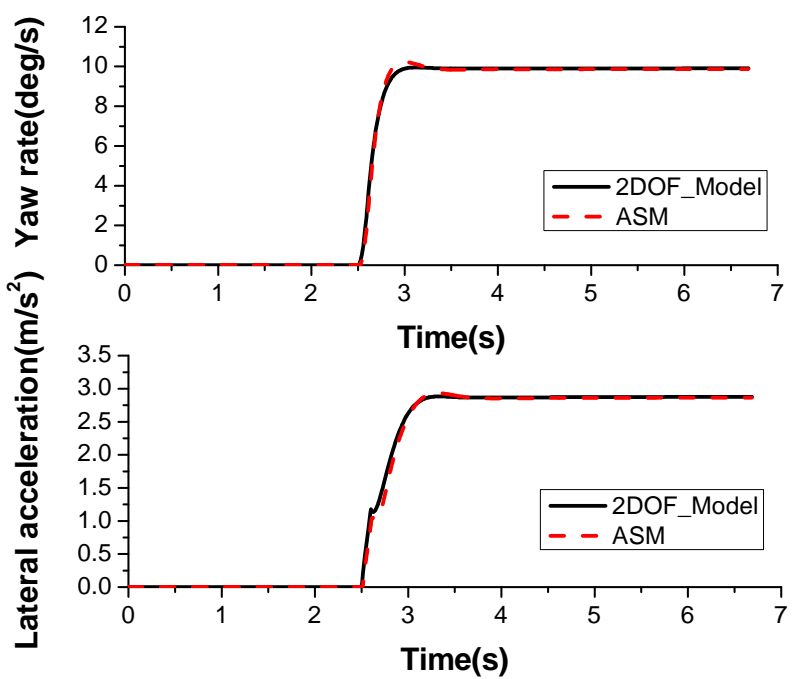

Figure 5. Steering wheel angle step input simulation $(60 \mathrm{~km} / \mathrm{h})$ 




Figure 6. Steering wheel angle step input simulation $(100 \mathrm{~km} / \mathrm{h})$



Figure 7. Slalom test simulation $(70 \mathrm{~km} / \mathrm{h})$

As can be seen from all above figures, yaw rate of the 2-DOF vehicle model is quite consistent with ASM when vehicle tire lateral forces are in linear region.

\section{Conclusions}

The simulation results compared with high-precision dynamic simulation model show that, the equivalent tire cornering stiffnesses of 2-DOF vehicle model's front and rear axles can be calibrated with fminsearch method simply and effectively, and it makes the yaw rate calculated from 2-DOF vehicle model reliable as the driver desired ideal value in ESP.

\section{References}

[1] Anton T. VAN ZANTEN, Rainer ERHARDT, Georg PFAFF, Friedrich KOST, Uwe HARTMANN, Thomas EHRET: Control Aspects of the Bosch-VDC, SAE Paper, 9624225 (1996).

[2] NHTSA. Federal Motor Vehicle Safety Standards No.126: Electronic Stability Control Systems (2007), http://www.nhtsa.gov/Laws+\&+Regulations/Electronic+Stability+Control+(ESC).

[3] Thomas D. Gillespie: Fundamental of Vehicle Dynamics, (2006).

[4] Information on MATLAB Help Document.

[5] Guillaume BAFFET, Joanny STEPHANT, Ali CHARARA: Vehicle Sideslip Angle and Lateral Tire-Force Estimations in Standard and Critical Driving Situations: Simulations and Experiments, AVEC'060026 (2006).

[6] A.T.van Zanten, Bosch ESP System: 5 Years of Experience, SAE Paper, 2000-01-1633 (2000).

[7] Taehyun Shim and Donald Margolis: Using $\mu$ Feedforward for Vehicle Stability Enhancement, SAE Paper,2000-01-1634 (2000). 